\title{
NOISE IN SENSOR CRYSTALS: HERMETICALLY SEALED AND UNSEALED
}

\author{
Yoonkee Kim and John R. Vig, US Army CECOM, Fort Monmouth, NJ
}

\begin{abstract}
Noise limits the accuracy with which the frequency of an oscillator can be determined. Similarly, in a resonant sensor, noise limits the measurement resolution, i.e., it limits the minimum quantity of a measurand that can be measured with a specified uncertainty. In some sensor applications, such as in quartz crystal microbalances (e.g., chemical sensors), the sensor crystals are not hermetically sealed.

The noise $\left[\sigma_{y}(\tau)\right.$ at several $\left.\tau\right]$ of hermetically sealed 5 $\mathrm{MHz}$ fundamental mode AT-cut quartz resonators was measured at laboratory ambient temperature, and in an oven near the turnover temperatures $\left(\sim 83^{\circ} \mathrm{C}\right)$. Then the hermetic seals were broken and the resonators were measured again at ambient and turnover temperatures. As one would expect, breaking the hermetic seal increased the equivalent series resistance and the aging of the resonators. When the resonators were in the oven near their turnover temperatures, the unsealed resonators' (drift-removed) short-term stability was not significantly different from that of the sealed ones. In fact, in some cases, the unsealed resonators exhibited better short term stability. The noise due to temperature fluctuations at laboratory ambient temperatures dominated the effects of breaking the hermetic seal.
\end{abstract}

\section{Introduction}

There exists a large amount of literature on the shortterm stability of hermetically sealed quartz resonators. For example, a search of the UFFC Digital Archive, www.ieee-uffc.org/archive, on "short term stability" or "short-term stability" and "quartz resonator" resulted in "6,196 results found". (Some of these results are not relevant, but, nevertheless, there are many papers available on the subject). We were unable to find papers that would answer the question of how the noise is affected when the hermetic seal of a (BAW) crystal is broken and the crystal is exposed to air.
Parker $[1,2]$ showed that when SAW delay line "devices are hermetically sealed in a clean package, the noise level remains high. If the device is operated in an unsealed package, the noise level decreases with time. The decrease in noise is even more dramatic if the package contains a small amount of silicone rubber...If a treated SAW device is UV-cleaned, the noise level can be increased again to its original value." [1]

It has been shown theoretically that the adsorption and desorption of molecules, more specifically, the fluctuations in the number of adsorbed molecules, can affect the short term stability of, especially high frequency, resonators $[3,4]$.

\section{Measurement Setup}

We selected several resonators from a batch of $5 \mathrm{MHz}$ fundamental mode AT-cut resonators that had been sealed in glass packages more than 30 years ago. The resonator blanks were $14 \mathrm{~mm}\left(0.550^{\prime \prime}\right)$ diameter, plano-convex, polished, with $3.3 \mathrm{~mm}$ diameter pure gold (no adhesion layer) electrodes, and a two-point mount. The turnover temperatures were $\sim 83^{\circ} \mathrm{C}$. Figure 1 shows the typical frequency vs. temperature (f vs. T) characteristics. Because the resonators were fundamental mode, and the Qs were low compared to the $\mathrm{Q}$ of a $5 \mathrm{MHz}$ state-of-theart overtone resonator, the short term stabilities were also lower than the state-of-the-art at $5 \mathrm{MHz}$.

Figure 2 shows a schematic diagram of the short-term stability measurement setup. When measured at the turnover temperature, the resonator was in an oven controlled crystal oscillator (OCXO) that had been designed especially for testing resonators [5]. The resonance frequency was $5 \mathrm{MHz}$ at the turnover temperature, as was the reference oscillator, so a low noise detuning capacitor of $33 \mathrm{pF}$ was connected in series to a terminal of the resonator in order to get conveniently measurable beat frequencies via a mixer. The beat frequency was typically +500 to $700 \mathrm{~Hz}$, depending on the temperature. The beat frequency was measured using a frequency counter (HP53132A) with gate times of $\tau=$ 


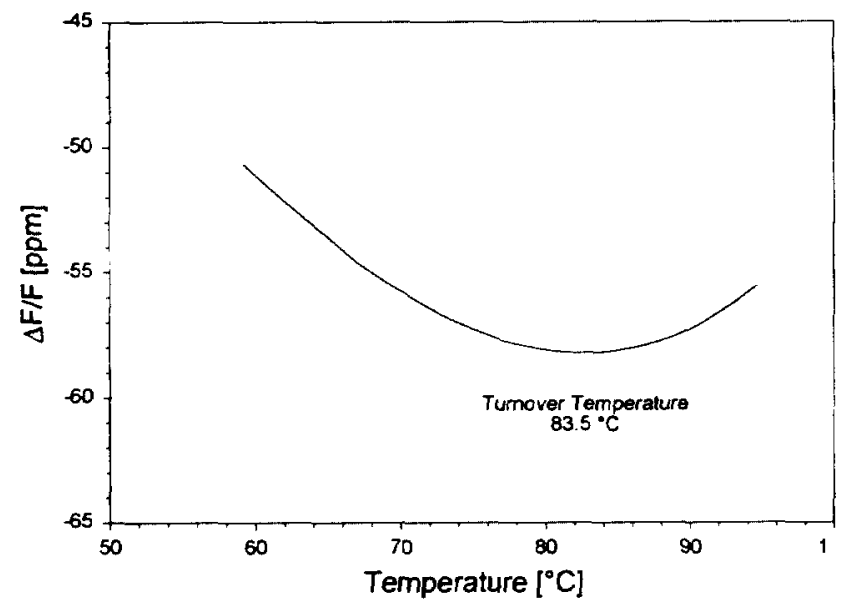

Figure 1: Typical frequency-temperature curve of the $5 \mathrm{MHz}$ fundamental mode AT-cut resonators investigated. The turnover temperature is at $83.5^{\circ} \mathrm{C}$.

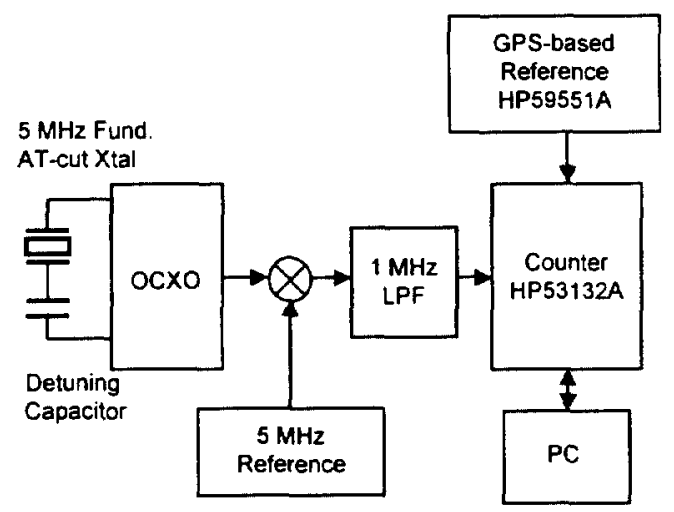

Figure 2: Measurement setup.

$0.1,0.2,0.5,1,2$, and 5 seconds. The frequency counter utilized a reference frequency source locked to the global positioning system (GPS) for its time base (HP59551A). The dead time was $\sim 0.1$ second. For each gate time, the sample size was 1000 . Thus, it took $\sim 2.6$ hours to collect the whole set of data for a short-term stability curve.

The resonator was on a workbench during the room temperature measurements, and in a temperature controlled thermos bottle during measurements at the turnover temperature.

The AC current through the resonator was measured using a miniature current probe.

\section{Results and Discussions}

Table 1 shows the equivalent circuit parameters of the resonators before and after breaking the seal, measured using an impedance analyzer (HP4194A). As might be expected, the motional resistance $R_{1}$ increased upon breaking the seal while the other parameters remained approximately unchanged.

Table 1: Equivalent circuit parameters.

\begin{tabular}{|c|c|c|}
\hline $\begin{array}{c}\text { Equivalent } \\
\text { circuit } \\
\text { parameters }\end{array}$ & Sealed & Unsealed \\
\hline $\mathrm{R}_{1}[\mathrm{ohms}]$ & $4.1 \sim 5.8$ & $13.6 \sim 15.7$ \\
\hline $\mathrm{L}_{1}[\mathrm{mH}]$ & $91.7 \sim 103.5$ & $\sim$ same \\
\hline $\mathrm{C}_{\mathrm{I}}[\mathrm{fF}]$ & $9.8 \sim 11.1$ & $\sim$ same \\
\hline $\mathrm{C}_{\mathrm{o}}[\mathrm{pF}]$ & $2.7 \sim 2.8$ & $\sim$ same \\
\hline $\mathrm{Q}$ & $5.8 \sim 7.1 \times 10^{5}$ & $1.9 \sim 2.4 \times 10^{5}$ \\
\hline
\end{tabular}

Initially, the measurement sequence was as follows:

1) measure the sealed resonator at the turnover temperature,

2) measure the sealed resonator at room temperature

3) break the seal and measure the unsealed resonator at room temperature, and

4) measure the unsealed resonator at the turnover temperature.

The entire sequence was finished within $\sim 1.5$ days. The results are shown in Figures 3 and 4. Figure 3 shows the short-term stability of five sealed and unsealed resonators measured at room temperature. As the laboratory ambient temperature was $-60^{\circ} \mathrm{C}$ from the turnover temperature, where the $f \mathrm{vs}$. T slope was $\sim 1 \mathrm{ppm} /{ }^{\circ} \mathrm{C}$, the temperature fluctuations probably dominated the effects of breaking the seal; i.e., there is little difference between the results for sealed and unsealed resonators. A $5.115 \mathrm{MHz}$ reference $\mathrm{OCXO}$ was also tested to verify that the setup's noise floor was sufficient. It was sufficiently low, i.e., $3 \times 10^{-12}$ for $\tau=5 \mathrm{sec}$.

Figure 4 shows the short-term stabilities at the turnover temperatures. The results show that the noise of unsealed crystals can be lower than the noise of hermetically sealed crystals. After collecting the data, we found that the automatic gain control (AGC) circuit board didn't function properly. The resonator currents were $\sim 4$ $\mathrm{mA}$ and $\sim 3 \mathrm{~mA}$ for the sealed and unsealed resonators, respectively. 


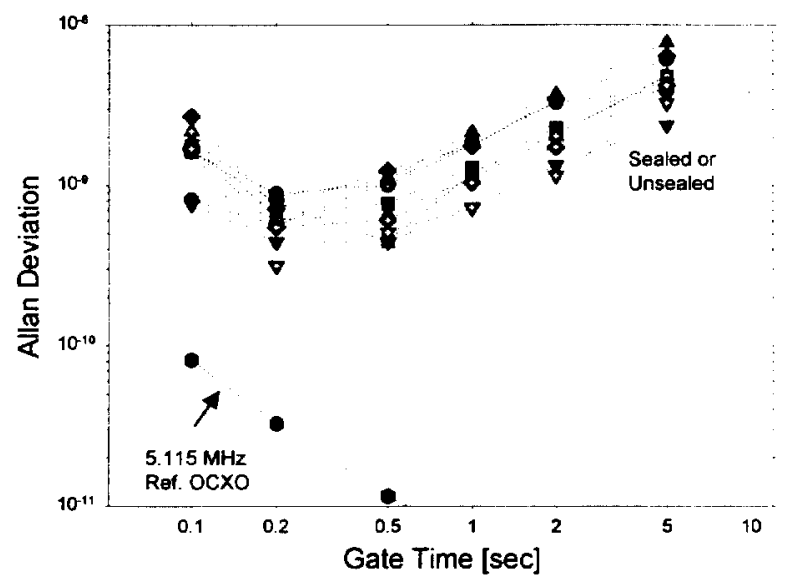

Figure 3: Short term stabilities at room temperature. Data for sealed and unsealed resonators are shown with solid symbols and + marked symbols, respectively.

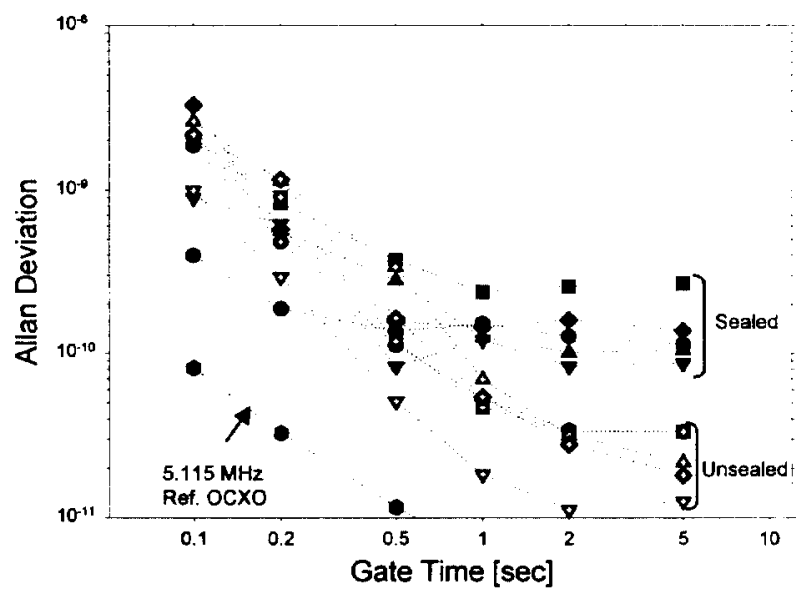

Figure 4: Short term stabilities at the turnover temperature.

After replacing the AGC board, the current was maintained at $\sim 0.15$ to $\sim 0.17 \mathrm{~mA}$ for both sealed and unsealed resonators. Figures 5 and 6 show the results with this current. At room temperature, we observed the same temperature-fluctuation-dominated behavior in both the sealed and unsealed resonators. Thus, further measurements were conducted at the turnover temperatures only.

The procedure used for obtaining the results shown in Figures 5 and 7 was as follows. Data collection started $\sim 2$ hours after turning on the oven, and it was completed $\sim 2.6$ hours later. The results are shown in curve 1 of Figure 5a. After 23 days of aging, the resonator was measured again - the results are shown in curve 2 of Figure 5a. The resonator enclosure was then broken. To remove resulting particles from the surfaces, the unsealed resonators were driven with a $\sim 7.5 \mathrm{~mA}$ current for about three minutes, using the impedance analyzer. The shortterm stability was measured after five hours of stabilization in the oven, and again $\sim 3.5$ days later. The results are shown in curves 3 and 4 of Figure 5a. During the aging, the frequency was sampled every 10 minutes with $\tau=1$ sec. The aging improved the short-term stability of both the sealed and unsealed resonators.

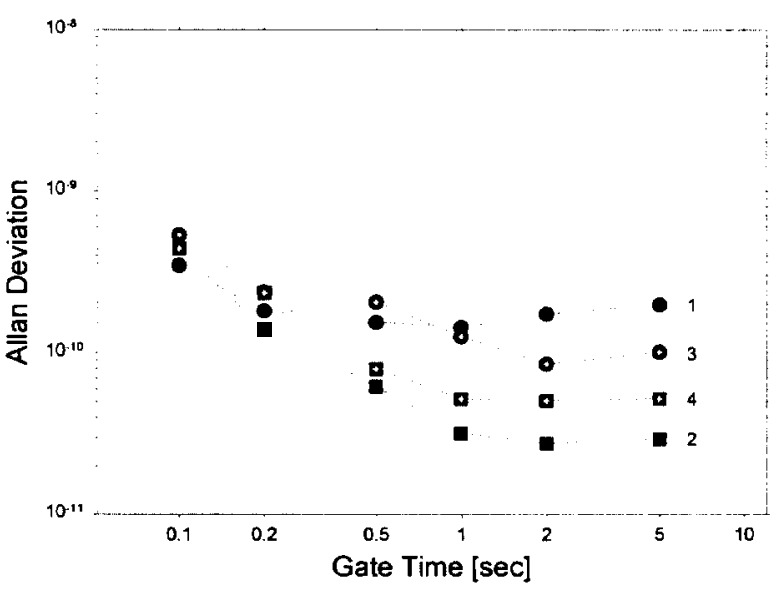

Figure 5a: Short-term stability of a resonator in oven.

1. sealed, within $\sim 5$ hours after turning-on oven,

2 . sealed, after $\sim 23$ days from 1 ,

3. unsealed, within $\sim 5$ hours after breaking seal and turning-on oven,

4. unsealed, after $\sim 3.5$ days from 3 .

Figure $5 \mathrm{~b}$ shows the results for another resonator. The trend is the same as the Fig. 5a case. The results for a third resonator were similar.

The effect of high driving an unsealed resonator (with $\sim 7.5 \mathrm{~mA}$ ) is shown in Figure 6. A sealed resonator was measured (curve 1), then its seal was broken, and it was measured again before and after the high driving (curves 2 and 3). The higher noise before high driving was accompanied by a higher equivalent series resistance, probably due to particles generated during opening the 


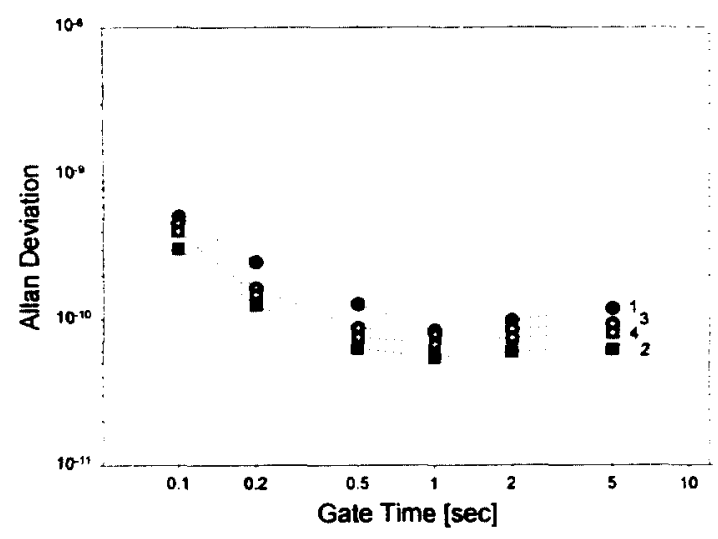

Figure 5b: Short-term stability of a resonator in oven.

1. sealed, within $\sim 5$ hours after turning-on oven,

2. sealed, after $\sim 3$ days from 1 ,

3. unsealed, within $\sim 5$ hours after breaking seal and turning-on oven,

4. unsealed, after $\sim 4$ days from 3 .

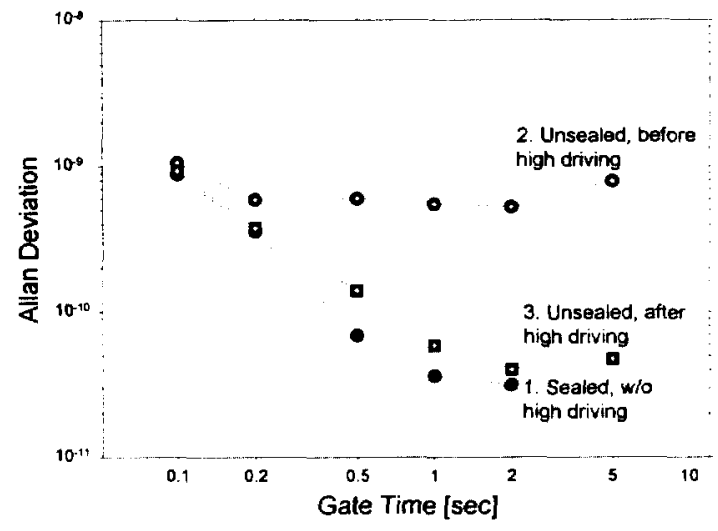

Figure 6: Effect of a high driving ( 7.5mA).

resonator enclosure, i.e., breaking the glass. High driving lowered both the resistance and the noise. The resistance was measured with an impedance analyzer (HP4192A). It was $\sim 22$ ohms before the high driving and $\sim 15$ ohms after. Shortly after the resistance dropped, the high driving was stopped.
Figure 7 shows the aging of two unsealed resonators during the -3.5 day period following the unsealing. Near the end of the period, the aging was about $-1.5 \times 10^{-7}$ /day, about $100 \times$ to $1000 \times$ higher than the aging of a similar sealed resonator after 3.5 days. The drift due to this high aging was removed during the calculations of short term stability.

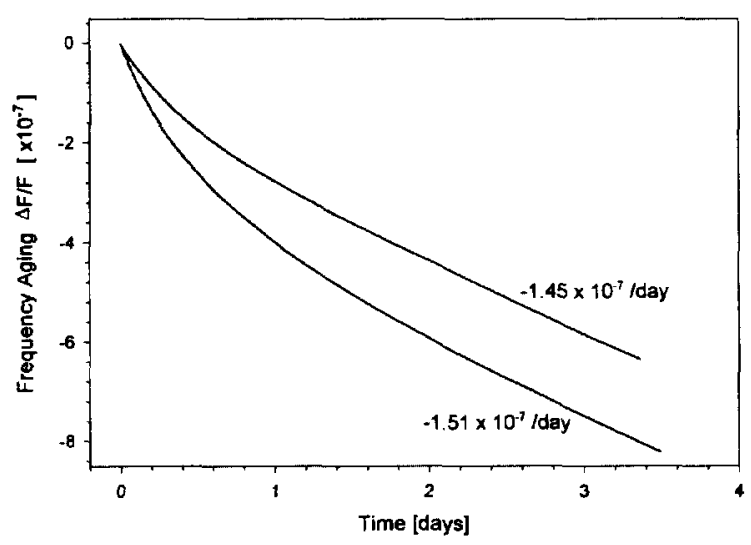

Figure 7: Aging of unsealed resonators for $\sim 3.5$ days. The aging data shown are during the last 24 hours.

\section{Conclusions}

The $\sim 100 \times$ to $1000 \times$ higher initial aging of an unsealed sensor crystal is an accuracy limitation (e.g., in a film thickness monitor that is regularly cycled between air and vacuum). As is the case for hermetically sealed resonators, the aging of an unsealed resonator is highest when the resonator is first heated to its turnover temperature. Similarly, the resonators' short term stability is higher shortly after the resonator is stabilized at its turnover temperature than it is after several days of aging.

Temperature fluctuation induced frequency changes dominate the short term stability when the temperature is far from the turnover temperature. The effect of breaking the hermetic seal is small, in comparison. The short term stability of an unsealed resonator at constant temperature was found to be comparable to, or lower than that of the same resonator when it was sealed.

Clean surfaces are known to rapidly adsorb contamination from the air. That air-exposed resonators can have better short-term stability than clean, hermetically sealed resonators agrees with previous 
results reported and mechanisms proposed in [1-4]. This result also suggests that adsorbed molecules affect the short-term stability, and that improvements in short-term stability may be achieved by means of an intentional deposition of, for example, an appropriate monolayer coating. High-driving a contaminated resonator can also improve the short term stability, probably because the high-driving removes particulate contamination.

\section{Acknowledgments}

The authors wish to thank V. J. Rosati, General Technical Services, Inc., Wall, NJ, and R. L. Filler, US Army CECOM, Ft. Monmouth, NJ, for suggestions on the measurement setup and helpful discussions.

\section{References}

[1] T.E. Parker, "1/f Phase Noise in Quartz Delay Lines and Resonators," Proc. 1979 Ultrasonics Symposium, pp. 878-881, 1979.

[2] T.E. Parker \& D.L. Lee, "Stability of Phase Shift on Quartz SAW Devices," Proc. 33 $3^{\text {rd }}$ Ann. Symp. On Frequency Control, pp. 379-387, 1979.

[3] J.R. Vig, "The Effects of Surface Contamination on the Noise and Drive-Level Sensitivity of Piezoelectric Resonators," Research and Development Technical Report SLCET-TR-87-5, US Army Laboratory Command, May 1987, AD-A181299.

[4] Y-K Yong \& J.R. Vig, "Resonator Surface Contamination - a Cause of Frequency Fluctuation?" IEEE Trans. on UFFC, vol. 36, pp. 452-458, 1989.

[5] H.W. Jackson, "Update on the Tactical Miniature Crystal Oscillator Program," Proc. Ann. Frequency Control Symp., pp. 492-498, 1982. 